\section{BARRIOS ALTOS. LA OTRA HISTORIA DE LIMA SIGLOS XVIII-XX ALEJANDRO REYES FLORES}

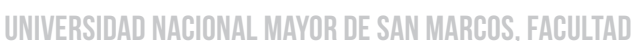
DE CIENCIAS SOCIALES, FONDO EDITORIAL, LIMA - PERÚ, 2015.

RESEÑA DE AARON URDANIGUE CONTRERAS

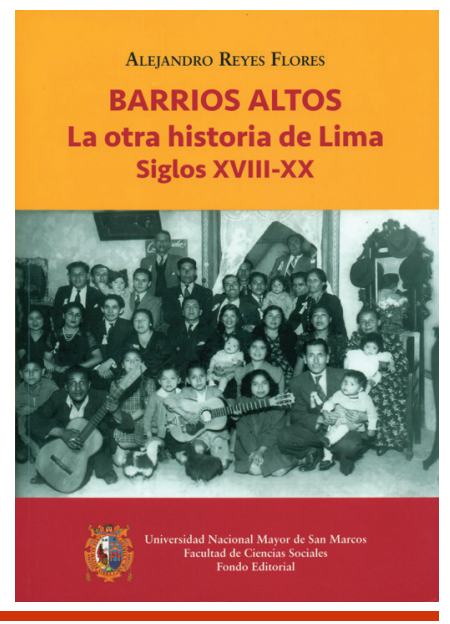

Esta publicación nace en el marco de una investigación académica para dar a conocer a los Barrios Altos, que para juicio del autor ha sido siempre subvalorada por no encontrarse en el centro del poder colonial y republicano. Es pertinente resaltar la filiación que existe entre el autor y los Barrios Altos, lugar donde vivió y que lo une emocionalmente con el área de estudio.

Si queremos investigar documentos y publicaciones referidos a los Barrios Altos, estos van a ser escasos, debido a que la mayoría de investigaciones se han centrado principalmente en el área que ha sido inscrita en la lista de Patrimonio Cultural de la Humanidad. Sin embargo, se dejó de contar la historia de lo que pasaba en las áreas periféricas de Lima colonial y republicana como es el caso de los Barrios Altos.

Y es que ahí reside el valor de esta publicación, de resaltar al "anti-héroe" o al artista de papel secundario, ya que el libro nos hace fabulosos recuentos de los distintos actores sociales y económicos que se desarrollaron en este lado de la ciudad, quizás para muchos desconocidos, como el aguador, el boticario-farmacéutico, así como también los "maestros" albañiles, carpinteros, pintores y sastres, la mayoría de ellos de condición humilde y que gracias a su trabajo hacían posible la vida de las demás personas en la otra Lima.

Es abundante la información obtenida de archivos históricos e información oral, donde se da cuenta también de la existencia de estos otros lugares de uso común de la gente de Barrios Altos como eran: los cajones, chicherías, chinganas y pulperías, todas ellas ya desaparecidas.

Gráficamente la propuesta es limitada, contando con 334 páginas, dividido en diecisiete capítulos, y narrado principalmente desde el s. XVIII hasta mediados del s. XX, de las cuales pasamos a describirlas en 6 partes:

En el primer capítulo describe geográficamente el entorno de Lima, así como su ocupación pre-hispánica y la inicial presencia ibérica en el valle, igualmente hace una descripción de la temprana fisonomía de Barrios Altos.

En el segundo bloque están comprendidos los capítulos II, III y IV, que describen la vida económica de los monasterios y conventos de los Barrios Altos. Asimismo, nos cuenta sobre las decisiones que tuvieron que tomar las familias pertenecientes a la nobleza limeña en momentos difíciles como fue el cambio y transición del gobierno colonial al republicano. Del mismo modo cabe mencionar el aporte del famoso icono de la arquitectura industrial de los Barrios Altos, como fue el Molino de Santa Clara.

El tercer bloque abarca los capítulos $\mathrm{V}$ y VI, de los cuales narra la presencia italiana a lo largo del s. XIX. Asimismo, describe la presencia del inmigrante chino, sufrida en un inicio pero gracias a su emprendimiento lograron posicionarse con pulperías, chinganas, fondas y en el tema cultural con los teatros.

En el cuarto bloque compuesto por los capítulos VII, VIII, IX y X, describe las actividades, económicas, mercantiles, manufactureras y de servicios de los Barrios Altos. Nos revela a los albañiles, carpinteros, pintores y sastres, y como se agrupaban en gremios y cofradías. Del mismo modo el autor presenta abundante información de los locales comerciales ya desaparecidos de chicherías, chinganas, pulperías y cajones, y el no menos conocido como la Plaza de Abastos de Lima. Asimismo, las industrias de la época como las curtiembres, velerías y panaderías, ubicadas la mayoría de ellas en los alrededores del rio Huatica, para aprovechar su fuerza hidráulica. Igualmente, el autor nos explica como prácticamente existió una ciudad universitaria con la presencia de la Facultad de Medicina de San Fernando y los hospitales de Santa Ana y San Bartolomé en los alrededores de la plaza Santa Ana de los Barrios Altos.

Del quinto bloque comprendido por los capítulos XI y XII, se desprende las transformaciones que sufrió Barrios Altos por efectos de la modernización de inicios del s. XX, con la presencia del tranvía, los cinemas, el "entierro" del rio Huatica, el trazado de nuevas calles y la construcción de las novedosas quintas. Además, la presencia del migrante japonés, y el aporte que tuvo en nuestra gastronomía.

Como sexto y último bloque, comprendido por los capítulos del XIII al XVII, el autor nos describe sobre una de las características más importantes de los Barrios Altos, que es la música y el crioIlismo. Hace un recorrido sobre la formación del vals criollo, músicos relevantes, el aporte del ejército con las famosas retretas y la consolidación del vals criollo. Personaje a destacar es el compositor Luis Dean conocido como "el payador", él encarna lo que debió, debe y deberá ser un criollo de verdad.

Dicho esto, solo cabe invitarlos a sumergirse en una buena investigación sobre uno de los barrios de mayor tradición limeña, hoy en día olvidado y desconocido por muchos, pero que nos da base suficiente para valorar y rescatar lo que aún queda en los Barrios Altos. 
Templos virreinales de los valles de Lambayeque es una publicación de notos autores, arqueólogos por la Universidad Nacional de Trujillo; además, el mencionado por último, es dominico y de la Universidad San Martín de Porres. Investiga archivos nacionales, extranjeros y los aportes de Domingo Angulo (1920); Harth-Terré (1965), quien en 1941 hizo levantamientos en Saña; Víctor Pimentel, quien trabajó allí (1967); Lorenzo Huertas; Jorge Izquierdo; Antonio San Cristóbal; etc. Comprende cuatro capítulos: el primero, "La evangelización en los valles de Lambayeque", explica ese proceso regional; el segundo, "Las Cofradías lambayecanas", analiza 'reducciones' nativas y su calo poblacional, listando este núcleo económico, de exclusión social étnica y de evangelización, con sus 'ramadas'.

Ambos capítulos restantes describen la historia constructiva de templos, capillas y oratorios. El tercero, "Iglesias de la villa y pueblo de Saña", inicia desde los primeros asentamientos franciscanos; la iglesia de Nuestra Señora del valle de Chiclayo; el Convento de Santa María; la Matriz de Chiclayo; y la abandonada villa de Saña, para españoles no-encomenderos, con restos gótico-isabelinos y renacentistas en las iglesias Matriz, San Francisco, San Agustín y La Merced, y sus claustros. Menciona el hospital de San Juan Bautista y Santa Lucía, la iglesia periférica para indios. Prosigue con San Ildefonso de Chérrepe, reducción trasladada a Lagunas con restos y espadaña; San Francisco de Mócupe (Mócupe viejo), iglesia ruinosa; y la Matriz de San Pedro de Lambayeque con sus retablos: Nuestra Señora de la Merced de 1784-96 con cariátides y hermes, el llamado "retablo de los santos dominicos" de "ejecución netamente imperita" (p. 150) según San Cristóbal, que los autores descubren ser de 1923 y con documento de 1816 que no era de 'santos dominicos'. Escriben: "el padre San Cristóbal afirma que el artesonado mudéjar es posterior a 1600, y que antes solo habría habido cubiertas efímeras, la descripción [localizada por estos autores, de 1566, sin embargo] nos presenta un artesonado mudéjar muy bien elaborado" (p. 137). Detrás de la iglesia matriz, las iglesias para sus parcialidades presentan una arquitectura muy diferente: Santa Catalina; Santa Lucía; San Roque; y San Pedro, llamada desde 1890 capilla de San Francisco, donde doctrinaban desde que Lambayeque ocupó su actual ubicación,

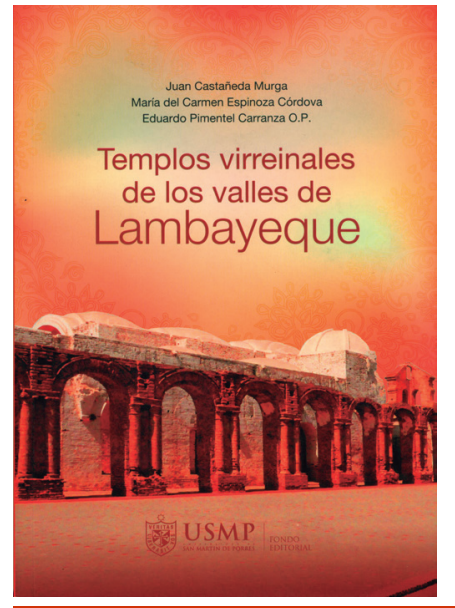

\title{
TEMPLOS VIRREINALES DE LOS VALLES DE LAMBAYEQUE
}

\author{
JUAN CASTAÑEDA, MARÍA DEL CARMEN ESPINOZA \& \\ EDUARDO PIMENTEL \\ UNIVERSIDAD SAN MARTÍN DE PORRES. FONDO EDITORIAL DE \\ LA UNIVERSIDAD SAN MARTíN DE PORRES, LIMA - PERÚ, 2015.
}

RESEÑA DE LEONARDO MATTOS-CÁRDENAS

después del fenómeno del Niño de 1578. Sigue San Pedro de Mórrope con sus arbotantes, iniciada en 1536 como reducción de pescadores con capilla abierta o 'guairona', que hoy luce capilla doctrinal de horcones y altar a pirámide escalonada, lo cual refleja sincretismo prehispánico y tradición: cuenta con yesos, mangles, algarrobos, columnas, horcones, y techados frecuentes con paja y estiércol.

La "madera de Guayaquil" Ilegaba de Chérrepe, Paita y Huanchaco, y para la iglesia de San Martín de Tours en Reque se empleó "madera de roble competente" (p. 164) en 1782 en "muy buen estado". Describen Santa María Magdalena de Eten, ruinas de la capilla que construyera Manuel del Castillo -terminada en 1778-; San Pedro de Monsefú; La Limpia Concepción de Mochumí; los restos de San Pedro de Túcume (Túcume viejo); la iglesia parroquial de Santa Lucía de Ferreñafe, con clásica portada lateral que San Cristóbal mostraba en clases que compartí (2005-6); la inconclusa iglesia de Santo Domingo de Olmos; San Julián de Motupe, dependiente en lo eclesiástico de Quito; San Pedro de Penachí; la iglesia anexa de San Pablo de Incahuasi; San Francisco de Salas, iglesia de la serranía; y San Juan Bautista de Cañaris (topónimo ecuatoriano).

El último capítulo, "Las capillas y oratorios de las haciendas" (de trigo, azúcar, panllevar, luego ingenios, trapiches, tinas), analiza San Juan de la Punta, única capilla exenta, ruinosa, edificada a inicios de 1900 con columnas entorchadas en yeso, que contaba con oratorios hoy desaparecidos: Santa Inés y San Antonio de Calupe, San Francisco Javier de Tumán, San Ildefonso de Picsi, Santa Maria de Sarrapo, San Agustín de Cojal, San Agustín de la Viña, Luya, San Juan Bautista de Chumbenique, San Francisco de la Otra Banda, el Salitral de la Soledad de Sicán y San Jacinto de Úcupe.

El libro termina con un "Glosario", y recoge alarifes inéditos, como hicieran Vargas Ugarte, Harth-Terré y San Cristóbal, quienes mostraban sus fichas sueltas. Unir estas contribuciones (vía web) sería loable.

Merecía mayor esfuerzo, sin embargo, la edición: la ANC (pp. 132, 133, 134) y la BM (pp. 117, 118, 121, 129) faltan entre las "Siglas y abreviaturas empleadas" ( $p$. 17); la Foto 27 está en posición incorrecta (p. 158); el "Plano de la destruida ciudad de Santiago de Miraflores de Saña" (p. 102) del obispo Martínez necesitaba mayor legibilidad y tamaño. Faltaría el "Plano del pueblo de Lambayeque" del mismo obispo, que creemos grafica bien las Iglesias 'étnicas' detrás de su Matriz.

Saña, con iglesias y claustros en ruinas pese a las intervenciones (2004) -donde según los autores "la incuria y una modernidad mal entendida han contribuido a su desaparición"--, y carente de museos, hace preguntarnos: ¿Sus célebres restos arquitectónicos (consolidados) pueden integrarse a su volumetría modular (documentada) faltante usando materiales ligeros antisísmicos? Ello detendría su deterioro, para así preservar patrimonio local colonial y prehispánico existente entre verde y tramas viales hoy ocultas. Se trataría de una operación simple, descentralizada, que alejaría eventuales protagonismos proyectuales. 


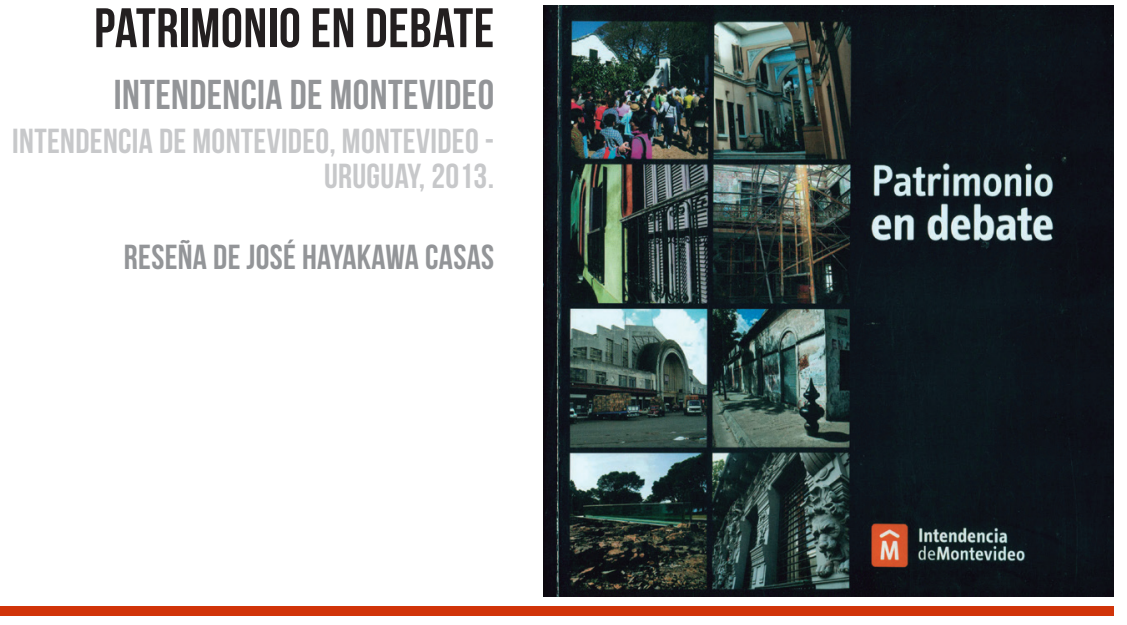

La oportunidad de reseñar este libro me permite abundar detalles sobre el valioso aporte de diversos académicos, actores sociales comprometidos con 'lo patrimonial', instituciones públicas vinculadas a la temática, distintos 'hacedores del patrimonio'y, también, de sus divulgadores en Uruguay, por reunirse, por debatir, por cuestionar, por reflexionar y por proponer en torno a buena parte de la acción en el patrimonio, la ciudad y las políticas públicas que vienen ocurriendo en la ciudad-departamento de Montevideo en los últimos 30 años, todo en el contexto del ciclo El Patrimonio en Debate, el cual se desarrolló entre marzo y agosto de 2012. Justamente la celebración de este referido ciclo brinda la oportunidad, el título y las ponencias que componen este valioso libro. Las actividades se desarrollaron en las salas del Teatro Solís entre el 22 de marzo y el 30 de agosto de 2012.

La presente publicación (226 páginas de extensión) incluye como principal contenido una trascripción del referido ciclo, de acceso libre, el cual fue desarrollado a través de seis mesas redondas y tres conferencias que reunieron invitados nacionales y extranjeros, así como la relatoría de cada una de las primeras. Los aspectos nucleares de este ciclo se recogieron en el libro del mismo nombre, aunque por razones de espacio las transcripciones sufrieron algunos ajustes, procurando mantener con un texto coherente, de forma que resultase lo más significativo y representativo de los conceptos allí vertidos. El evento y la publicación de este libro de ponencias fue producto del liderazgo -y preclaridad-del reconocido arquitecto Francisco Bonilla, y a su vez fue organizado por encargo e iniciativa de la Intendencia de Montevideo, con el apoyo de connotadas entidades uruguayas como el Ministerio de Educación y Cultura, la Comisión del Patrimonio Cultural de la Nación y la Facultad de Arquitectura de la Universidad de la República, además del auspicio de UNESCO.

Una de las principales misiones de dicho evento y la consiguiente publicación, además confrontar opiniones disímiles sobre el patrimonio uruguayo -en general-y montevideano -con especial atención-, y su adecuada gestión, incluir una visión más amplia, poliédrica, de un asunto en el que tradicionalmente han participado activamente pocas disciplinas. Ello es además muy pertinente debido al contexto local uruguayo, donde la actualización normativa -clamada y reclamada desde diversos sectores- tendría que incorporar las ideas que de estas visiones múltiples e interdependientes surgen.

En la apertura de la publicación la ponencia del arquitecto Juan Pedro Urruzola, quien desarrolla la línea argumental de cómo la planificación, la ciudad y el patrimonio son temáticas concurrentes y cada vez más complementarias. Por ello él plantea la necesidad de recordar que la ciudad es un organismo vivo y en construcción permanente, y que el presente representará en el futuro la memoria de dicha ciudad. De acuerdo a dicha concepción, el rol del patrimonio resulta clave porque debe participar en la configuración del presente y futuro urbanos. En ese marco, el autor reconoce el valor de estas jornadas para profundizar y actualizar las políticas patrimoniales de la Intendencia de Montevideo, y fortalecer un proyecto colectivo cuyo fin ulterior es construir un territorio más democrático, integrado y justo. También en la apertura, el arquitecto Mario Arana toma una bandera diferente: que la observación de las indecisiones, inconsecuencias $y / o$ insensibilidades obligan a la reflexión, a la toma de conciencia individual y colectiva, y a un sinceramiento institucional que apueste por ampliar la postura y la actuación.

Asimismo, destaca la Mesa 3, "La escala de lo patrimonial", en la cual no solo se discutió acerca de la evolución rastreable desde el reconocimiento de áreas caracterizadas, y su posterior protección mediante la creación de las Comisiones Especiales Permanentes y las normas respectivas, sino sobre otros sectores de la ciudad con valores derivados de su coherencia tipo morfológica y de sus rasgos identitarios, reapropiados socialmente. Las actuaciones de las referidas comisiones en Montevideo -comprendidas desde la perspectiva de la Planificación Territorial y Derivada- expresaron una visión sistémica que ya estimaba estos otros sectores en su valor de conjunto, más que como sumatoria de edificios notables y con méritos individuales a proteger. Dichos conjuntos urbanos pueden poseer calidades asociadas a lo monumental-nacional, o solo contar con valor simbólico y referencial para la comunidad que los habita.

Finalmente, en la Mesa 5, "Los hacedores del patrimonio (II)", se destacaron argumentos tangibles y verificables en favor de una recuperación patrimonial real y viable que se opone a la mirada catastrofista de los impedimentos tecnológicos o las deficiencias de capacitación. Estas razones alentadoras se nutren del reconocimiento de una arraigada tradición y savoir faire constructivo expresados no solo en los edificios monumentales sino en aquellos más modestos y autoconstruidos. Como demuestran las experiencias discutidas, si a ello se le suma el sentido común, la creatividad, la recuperación de oficios, y el aporte de nuevos productos y técnicas, su validez se potencia exponencialmente.

Dicho esto, solo cabe reiterarles la invitación convencida a sumergirse en el amplio espectro de lo patrimonial desde el debate uruguayo, donde la fulgurante aparición de este libro no cerró la discusión sino que, por el contrario, terminó de abrirla. 
En julio de 2016 aparece en el panorama de publicaciones científicas la revista Diferents. Revista de museus, que busca ser un espacio de reflexión en torno a la museografía. La edición corresponde al Museo de Arte Contemporáneo Vicente Aguilera Cerni de Vilafamés (MACVAC), en España, actualmente bajo la dirección de Xavier Allepuz. Su nombre refiere una vocación particular por visibilizar y difundir la existencia de museos que por sus características podrían considerarse singulares, tanto a nivel nacional como internacional.

El primer número se centra en el MACVAC, institución promotora de la publicación y, claramente, un espacio diferente en tanto museo de arte contemporáneo, dado que se trata de uno de los más antiguos dedicados a la muestra de nuevas tendencias artísticas en España. La edición, muy cuidada estéticamente, como corresponde a una revista dedicada mayoritariamente al arte, sigue un orden lógico en los contenidos: comienza con un recorrido por las diferentes concepciones de cómo debe ser una institución museística a lo largo del tiempo y el espacio, texto a cargo de Lola Jiménez (profesora titular de Historia del arte de la Universidad Complutense de Madrid); y se adentra después en el caso concreto que ocupa este primer número, que se trata desde muy variadas perspectivas. Por ejemplo, Vicent Àlvarez (Consejo Valenciano de Cultura) analiza las repercusiones socioeconómicas que supuso para la población de Vilafamés la creación del museo y, sobre todo, la incidencia que tuvo en la conservación y rehabilitación del conjunto histórico de la localidad, que culminó con su declaración como Bien de Interés Cultural. Naturalmente se hace hincapié en la colección, que analizan Jaime Millás (periodista y escritor) y Patricia Mir (crítica de arte), así como en la complejidad de la conservación y restauración de las obras de arte contemporáneo -artículo de María Teresa Pastor (restauradora)- y la gestión del fondo documental para la investigación, que abordan Lledó Felip, Mónica Fornals y Celina Torlá (documentalistas). La revista rinde también homenaje al crítico de arte fundador de la institución, Vicente Aguilera Cerni, a través del artículo escrito por su nieta, Mercedes Torres-Aguilera (editora), y el análisis de su carrera profesional realizado por Juan Ángel Blasco (Cate-

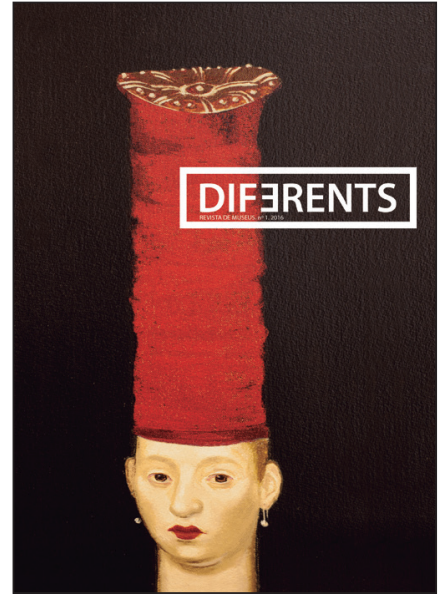

DIFERENTS.

REVISTA DE MUSEUS, 1, [DEDICADA A "MUSEO DE ARTE CONTEMPORÁNEO VICENTE AGUILERA CERNI" [MACVAC)]

\author{
MUSEO DE ARTE CONTEMPORÁNEO VICENTE \\ AGUILERA CERNI DE VILAFAMÉS
}

MUSEO DE ARTE CONTEMPORÁNEO VICENTE AGUILERA CERNI DE VILAFAMÉS, VILAFAMÉS - ESPAÑA, 2016.

RESEÑA DE JOAN FELIU FRANCH drático de Historia del Arte de la Universidad Politécnica de Valencia).

Esta revista redunda en que la idea de museo como simple depósito de materiales y centro de investigación reservado a una minoría ha dado paso a una concepción de la institución como un núcleo de proyección cultural y social, con una continua y decisiva función didáctica. Es más, de ahí el interés por reseñar esta bellísima publicación en Devenir; al igual que en el pasado las ciudades contendían por la realización de arquitecturas que eran la manifestación del poder, como una catedral o un palacio, en la actualidad asistimos a la necesidad de la imagen que ofrecen los museos como símbolo de identidad, de autenticidad, de las ciudades que los contienen. Ejemplos como el Guggenheim de Bilbao en España, el de Antioquía de Medellín en Colombia o el propio MACVAC lo evidencian.

Tradicionalmente, el impacto de un museo en una comunidad se inscribe concretamente en la posibilidad de conservación y acceso a obras de arte o a objetos de valor histórico, con fines de aumentar los conocimientos o la experiencia sensible, pero un museo debe concebirse también como una entidad que tiene oportunidad de transformar el entorno donde se encuentra.

¿Puede un museo reactivar la maltrecha economía de una ciudad? ¿Debería hacerlo? ¿No estaría desvirtuando su cometido principal, el de la difusión de conocimiento? Esta revista, enfocada en lo singular, en lo diferente en el ámbito de los museos, servirá para que repense- mos los factores y condiciones que determinan el grado de éxito o fracaso de estas entidades de arte globales que se emplean como dinamizadores locales.

Cada vez es cada vez más frecuente el uso de la arquitectura como instrumento del place marketing, márketing de ciudades, para alcanzar efectos de visibilidad global, especialmente en proyectos de regeneración urbana. El trabajo de arquitectos de renombre internacional como Frank Gehry, Norman Foster, Renzo Piano, Santiago Calatrava, Rem Koolhaas o Zaha Hadid, por mencionar algunos, constituye siempre un punto llamativo o hasta controvertido en cualquier proyecto de regeneración de una población. Sus defensores arguyen que la arquitectura de firma ya garantiza, de facto, el éxito de la operación. Sus detractores, por el contrario, nos recuerdan que la arquitectura de renombre es a todas luces insuficiente para asegurar el éxito de la inversión y de su elevadísimo riesgo operativo. Encontraríamos ejemplos para contentar a ambas partes. También, en cierta forma, es ese el caso del MACVAC, situado en un rehabilitado palacio del siglo $X V$ que sirvió, como se explica en la revista, para recuperar todo un conjunto urbano de una pequeña población. En todo caso, no se debe olvidar que el éxito de un museo guarda estrecha relación con su capacidad de implicar a su entorno próximo.

Deseamos larga vida a una revista diferents, que cuenta con una multiplicidad de enfoques sobre la museografía que de seguro generará reflexión sobre las cuestiones mencionadas, y traerá nuevos e interesantes debates. 